\title{
Relationship between epicardial fat tissue thickness and breast arterial calcifications in premenopausal women is
}

\author{
Cennet Ylldız ${ }^{\mathrm{a}, *}$, Abdulmelik Yıldız ${ }^{\mathrm{b}}$, Ahmet Karakurt ${ }^{\mathrm{c}}$ \\ a Tekden Hospital, Istanbul, Turkey \\ b Nisantasi University, Istanbul, Turkey \\ c Kafkas University, Kars, Turkey
}

\section{A R T I C L E I N F O}

\section{Article history:}

Received 29 August 2016

Received in revised form 15 October 2016

Accepted 18 October 2016

Available online 21 October 2016

\section{Objective}

Epicardial fat tissue (EFT) is located between myocardium and visceral pericardium. It has the same embryologic origin with the mesenteric omental fat tissue. It is very close to the myocardium and shares the same microcirculation. ${ }^{1}$ EFT functions as a mechanical buffer against coronary arterial wave torsion, plays an important role in maintaining fatty acid homeostasis and is thought to contribute coronary artery remodelling. ${ }^{2}$ EFT secretes several proinflammatory cytokines such as angiotensin II, tumor necrosis factor- $\alpha$, monocyte chemotactic protein-1, interleukin-6, resistin and plays an active role in inflammatory and atherosclerotic processes. ${ }^{3,4}$

Breast arterial calcifications (BAC) are a type of medial calcification detected at mammography. Its prevalence ranges between $1 \%$ and $49 \%$ in female population. ${ }^{5}$ Age, race and ethnicity are the most important factors influencing the prevalence of BAC. ${ }^{6}$ BAC have been associated with hypertension, ${ }^{7}$ diabetes mellitus, ${ }^{7}$ coronary artery disease ${ }^{8}$ and are a risk marker for future cardiovascular disease events. ${ }^{9}$ Women with mammography-detected BAC are more likely to have coronary artery calcification which is an early sign of coronary artery disease. ${ }^{10}$

In this study starting with the possibility of existence of correlation between EFT thickness and BAC, we aimed to investigate the relationship between EFT thickness and BAC in premenopausal women.

is Conflict of interest: None declared.

* Corresponding author at: Konaklar mah, Org. Izzettin Aksalur cad, Oyak sitesi sok, 38 Blok Daire 2. Besiktas, Istanbul, Turkey.

E-mail addresses: firdevsi2010@gmail.com (C. Yıldız),drmelik@hotmail.com (A. Yıldız), karakurt38@hotmail.com (A. Karakurt).

Peer review under responsibility of The Society of Cardiovascular Academy.

\section{Methods}

Among 756 premenopausal women who underwent diagnostic mammography between November 2015 and March 2016, 171 of these premenopausal women enrolled in this prospective study. Clinical indication for mammography was either routine screening mammography for women with no presenting complaints or diagnostic mammography for women with suspicious findings on breast ultrasound. The study protocol was approved by the Medipol University Ethics Committee and all subjects gave informed consent prior to participation. The study group consisted of 80 women with mammography detected BAC and the control group consisted of 86 women without BAC (an example of BAC is shown in Fig. 1.) Women with hypertension, diabetes mellitus, hyperlipidemia, ischemic heart disease, congestive heart failure, thyroid dysfunction, anemia, renal or hepatic failure, stroke or other chronic medical disorders were excluded from the study.

Blood samples were collected from antecubital vein after an overnight fast. Serum total cholesterol (TC), triglycerides (TG), high-density lipoprotein cholesterol (HDL-C), low-density lipoprotein cholesterol (LDL-C), fasting glucose were analysed.

Each subject underwent a full-field digital mammographic examination using a Mammomat 3000 Nova equipment (Siemens AG, Germany). All mammograms had craniocaudal and mediolateral oblique images of the two breasts. All images were interpreted by an experienced radiologist who was blinded to the echocardiography results. BAC were described as deposits of parallel lines of calcium when the arterial wall was imaged longitudinally or as calcific rings when the arterial wall was imaged transversely. ${ }^{11}$

All the subjects underwent full transthoracic echocardiographic examination using Vivid 3 pro (GE Vingmed Ultrasound AS, Horten, Norway) in accordance with the recommendations of the American Society of Echocardiograpy. ${ }^{12}$ Left ventricular ejection fraction (LVEF) was measured by using Simpson rule. Epicardial fat tissue was identified as echo free space between myocardium and visceral pericardium and its thickness was measured perpendicularly on the free wall of right ventricle from parasternal long-axis and short axis views at end diastole by an experienced cardiologist who was blinded to the clinical results of the patients. ${ }^{13}$ The average of three measurements was used for statistical analysis. 


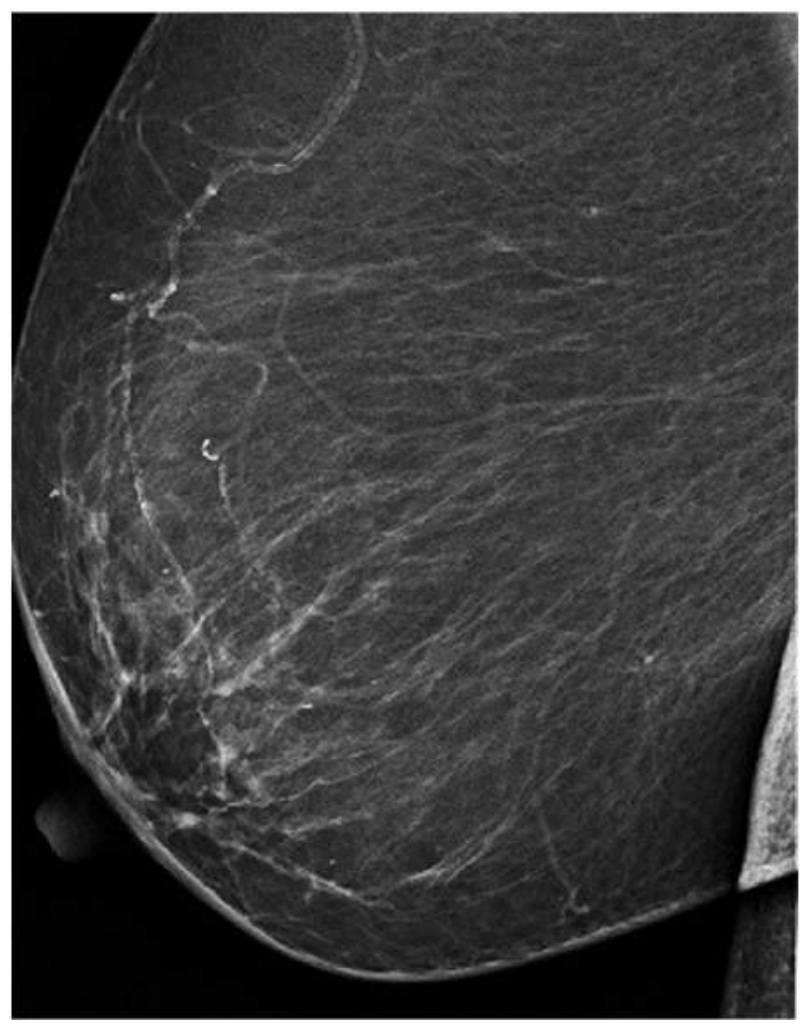

Fig. 1. An example of BAC.

\section{Statistical analysis}

Continuous variables were expressed as mean and standard deviation. Categorical variables were expressed as percentages. Parametric continuous variables and categorical variables were compared by Student's $t$-test and, the chi square test respectively. Multiple regression analysis was used to evaluate the possible associations of weight, BMI, glucose, LDL-C, HDL $-C$, TC, TG, EFT with BAC. $P$ values $<0.05$ were considered significant. All statistical studies were carried out by using the SPSS program (version 15.0; SPSS Inc., Chicago, Illinois, USA).

\section{Results}

Among 756 women who underwent screening and diagnostic mammography, 168 subjects met the entry criteria and were enrolled in the study. 80 women with BAC were accepted as the study group, and 86 women without BAC were accepted as the control group. No significant difference was detected between the two groups with respect to age, weight, height, BMI, LVEF, fasting glucose, TC, HDL-C and TG levels. LDL-C level and EFT thickness were statistically significantly higher in the study group compared to the control group $(3.65 \pm 0.73 \mathrm{~mm}$ vs. $2.94 \pm 0.67 \mathrm{~mm}, p<0.001$, respectively). Clinical and biochemical parameters of the patients are shown in Table 1 .

Multiple regression analysis showed that EFT thickness, TC, LDL-C and BMI were important associations of BAC (Table 2).

\section{Discussion}

Our study showed an association between EFT thickness and BAC in patients without structural heart disease. EFT thickness was significantly higher in patients with BAC. There was a strong association between EFT thickness and BAC. We also found positive associations between EAT thickness and TC, BMI, LDL-C.

Anatomically EFT, located between myocardium and visceral pericardium, is mainly concentrated in the atrioventricular and interventricular
Table 1

Clinical and biochemical parameters of the patients.

\begin{tabular}{llll}
\hline & $\begin{array}{l}\text { Study group } \\
(n=80)\end{array}$ & $\begin{array}{l}\text { Control group } \\
(n=86)\end{array}$ & $\mathrm{P}$ \\
\hline Age & $44.7 \pm 3.7$ & $45.1 \pm 3.3$ & 0.36 \\
Height $(\mathrm{m})$ & $1.64 \pm 0.08$ & $1.65 \pm 0.07$ & 0.37 \\
Weight $(\mathrm{kg})$ & $74.3 \pm 10.7$ & $72.8 \pm 9.2$ & 0.33 \\
BMI & $27.8 \pm 5.1$ & $26.8 \pm 4.1$ & 0.16 \\
Smokers, $\mathrm{n}(\%)$ & $15(18)$ & $18(20.4)$ & 0.44 \\
Alcohol, $\mathrm{n}(\%)$ & $8(9.6)$ & $9(10.2)$ & 0.57 \\
Fasting Glucose (mg/dl) & $99.7 \pm 10.3$ & $96.9 \pm 8.3$ & 0.06 \\
TC $(\mathrm{mg} / \mathrm{dl})$ & $190.7 \pm 36.7$ & $188.3 \pm 26.6$ & 0.64 \\
LDL-C (mg/dl) & $133.7 \pm 25.9$ & $125.1 \pm 27.8$ & 0.04 \\
HDL-C (mg/dl) & $43.4 \pm 9.8$ & $41.8 \pm 9.9$ & 0.30 \\
TG (mg/dl) & $157.2 \pm 58.9$ & $156.6 \pm 59.3$ & 0.94 \\
SBP (mmHg) & $131.4 \pm 11.6$ & $130.1 \pm 18.2$ & 0.59 \\
DBP (mmHg) & $81.8 \pm 8.7$ & $82.3 \pm 8.1$ & 0.72 \\
LVEF $(\%)$ & $62.7 \pm 4.1$ & $63.3 \pm 3.5$ & 0.36 \\
EFT thickness (mm) & $3.65 \pm 0.73$ & $2.94 \pm 0.67$ & $<0.001$ \\
\hline
\end{tabular}

TC: Total cholesterol; LDL-C: Low density lipoprotein cholesterol; HDL-C:High density lipoprotein cholesterol; TG: Triglyceride; SBP: Systolic blood pressure DBP: Diastolic blood pressure; LVEFF: Left ventricular ejection fraction; EFT: Epicardial Fat tissue.

grooves and shares the same microcirculation with the myocardium and coronary arteries. ${ }^{14}$ EFT has paracrine and vasocrine activities, have both harmful and protective effects on heart through release of variety of adipocytokines. ${ }^{15}$ Recently, it has been demonstrated that increased EFT thickness occurs with disorders such as hypertension,. ${ }^{16}$ insulin resistance, ${ }^{17}$ metabolic syndrome, ${ }^{18}$ and coronary atherosclerosis. ${ }^{4}$ Several studies have assessed the relationship between EFT and coronary artery disease. , $4,15,19$ The pathophysiological mechanisms responsible for CAD development through EFT are not clearly elucidated. In patients with CAD, inflammatory pathways within EFT are activated and EFT secretes more chemokines and cytokines that mediate inflammation. ${ }^{20}$ EFT may act as an endocrine organ by secreting various factors in the circulation thus contributing coronary lesion formation.

Vascular calcifications occur in the intima or media layers of the vessel wall. Intimal calcification is almost always associated with atherosclerosis, whereas BAC are a form of medial arterial sclerosis which is independent of atherosclerosis. ${ }^{21,22}$ These two types of calcification represent pathophysiological different mechanisms. Intimal calcification is related to chronic inflammatory changes which involve growth factors, inflammatory cells, lipid deposition and macrophage accumulation. ${ }^{21}$ Medial arterial calcification is almost always associated with aging, chronic renal failure or diabetes and involves the differentiation of vascular smooth muscle cells into osteoblast like cells. ${ }^{22}$ BAC are correlated with subsequent development of coronary artery calcifications, ${ }^{23-25}$ Kemmeren et al. found that presence of BAC was associated with a $30 \%$ and $90 \%$ increase in cardiovascular mortality in non-diabetic and diabetic women, respectively. ${ }^{26}$ Rotter et al. showed that mammographically detected BAC was associated with an increased prevalence of cardiovascular risk factors and cardiovascular morbidity. ${ }^{11}$ A recent study has shown that presence of BAC are stronger risk factor than other cardiovascular risk factors such as diabetes, hypertension and hyperlipidemia for coronary artery calcification. ${ }^{27}$

Until several decades ago, it had been thought that coronary artery disease was primarily a man's disease. Today, it is recognised that this

Table 2

Parameters that have associations with BAC.

\begin{tabular}{llll}
\hline & OR & $95 \%$ C.I. & P \\
\hline TC & 0.962 & $0.938-0.986$ & 0.002 \\
LDL-C & 0.955 & $0.924-0.987$ & 0.007 \\
BMI & 0.631 & $0.510-0.782$ & $<0.001$ \\
EFT thickness & 869.4 & $97.91-7719.8$ & $<0.001$ \\
\hline
\end{tabular}

BAC: Breast arterial calcifications; BMI: Body mass index; TC: Total cholesterol; LDL-C: Low density lipoprotein cholesterol; HDL-C: High density lipoprotein cholesterol; TG: Triglyceride. 
belief is not true, as cardiovascular disease is the most common cause of death among women throughout the world; In addition cardiovascular mortality rate among young women is still on the rise. Prevention and diagnosis of coronary artery disease are of great importance, because not only it increases the chances of cure, but it also reduces the major complications that could be avoided at an early stage. Traditional risk factors such as hyperlipidemia, hypertension, family history, diabetes mellitus and smoking are commonly used to assess the cardiovascular disease risk. Besides traditional risk factors, several new coronary risk factors have been identified and play significant roles in the atherosclerosis development. ${ }^{28,29}$ One of those risk factors is BAC. Recent studies have shown that presence of BAC on mammograms indicates a greater risk for coronary artery disease. ${ }^{27}$ As the EFT thickness was positively correlated with BAC and LDL-C levels, risk factors for coronary artery disease, in our study, EFT thickness measurement might play an important role in coronary artery risk stratification for premenopausal women in clinical practice.

\section{Study limitations}

Visual scoring of conventional mammography was applied in BAC assessment, the calcification mass scoring which is used at the digital mammography was not performed.

\section{References}

1. Fei J, Mazzoccoli G. Epicardial fat is an important visceral adipose depot influencing cardiovascular disease and metabolic syndrome. J Clin Exp Cardiolog 2013;4(3)e121.

2. Rabkin SW. Epicardial fat: properties, function and relationship to obesity. Obes Rev 2007;8(3):253-261

3. Fitzgibbons TP, Czech MP. Epicardial and perivascular adipose tissues and their influence on cardiovascular disease: basic mechanisms and clinical associations. J Am Heart Assoc 2014;3(2)e000582.

4. Yerramasu A, Dey D, Venuraju S, et al. Increased volume of epicardial fat is an independent risk factor for accelerated progression of sub-clinical coronary atherosclerosis. Atherosclerosis 2012;220(1):223-230.

5. Iribarren C, Molloi S. Breast arterial calcification: a new marker of cardiovascular risk? Curr Cardiovasc Risk Rep 2013:7(2):126-135.

6. Reddy J, Son H, Smith SJ, Paultre F, Mosca L. Prevalence of breast arterial calcifications in an ethnically diverse population of women. Ann Epidemiol 2005;15(5):344-350.

7. Cetin M, Cetin R, Tamer N, Kelekçi S. Breast arterial calcifications associated with diabetes and hypertension. J Diabetes Complications 2004;18(6):363-366.

8. Schnatz PF, Marakovits KA, O'Sullivan DM. The association of breast arterial calcification and coronary heart disease. Obstet Gynecol 2011;117(2 Pt 1):233-241.
9. Kemmeren JM, van Noord PA, Beijernick D, Frachebound J, Banga JD, van der Graaf Y. Arterial calcifications found on breast screening mammograms and cardiovascular mortality in women. Am J Epidemiol 1998;147(4):333-341.

10. Margolies L, Salvatore M, Hecht HS, et al. Digital mammography and screening for coronary artery disease. J Am Coll Cardiol Img 2016;9(4):350-360.

11. Rotter MA, Schnatz PF, Currier Jr AA, O'Sullivan DM. Breast arterial calcifications (BACs) found on screening mammography and their association with cardiovascular disease. Menopause 2008;15:276-281.

12. Gottdiener JS, Bednarz J, Devereux R, et al. American Society of Echocardiography recommendations for use of echocardiography in clinical trials. J Am Soc Echocardiogr 2004;17(10):1086-1119.

13. Iacobellis G, Assael F, Ribaudo MC, et al. Epicardial fat from echocardiography: a new method for visceral adipose tissue prediction. Obes Res 2003;11:304-310.

14. Sacks HS, Fain JN. Human epicardial adipose tissue: a review. Am Heart J 2007;153(6): 907-917.

15. Katsiki N, Mikhailidis DP, Wierzbicki AS. Epicardial fat and vascular risk: a narrative review. Curr Opin Cardiol 2013;28:458-463.

16. Dicker D, Atar E, Kornowski R, Bachar GN. Increased epicardial adipose tissue thickness as a predictor for hypertension: a cross-sectional observational study. J Clin Hypertens (Greenwich) 2013;15:893-898.

17. Iacobellis G, Leonetti F. Epicardial adipose tissue and insulin resistance in obese subjects. J Clin Endocrinol Metab 2005;90(11):6300-6302.

18. Yorgun H, Canpolat U, Hazırolan T, et al. Increased epicardial fat tissue is a marker of metabolic syndrome in adult patients. Int J Cardiol 2013;165(2):308-313.

19. Börekçi A, Gür M, Özaltun B, et al. Epicardial fat thickness in stable coronary artery disease: its relationship with high-sensitive cardiac troponin $\mathrm{T}$ and $\mathrm{N}$-terminal pro-brain natriuretic peptide. Coron Artery Dis 2014 Dec;25(8):685-690.

20. Mazurek T, Zhang L, Zalewski A, et al. Human epicardial adipose tissue is a source of inflammatory mediators. Circulation 2003;108:2460-2466.

21. Mackey RH, Venkitachalam L, Sutton-Tyrrell K. Calcifications, arterial stiffness and atherosclerosis. Adv Cardiol 2007;44:234.

22. Leopold JA. Vascular calcification: mechanisms of vascular smooth muscle cell calcification. Trends Cardiovasc Med 2015;25(4):267-274.

23. Hendriks EJ, de Jong PA, van der Graaf Y, Mall WP, van der Schouw YT, Beulens JW. Breast arterial calcifications: a systematic review and meta-analysis of their determinants and their association with cardiovascular events. Atherosclerosis 2015;239(1): $11-20$.

24. Maas AH, van der Schouw YT, Atsma F, et al. Breast arterial calcifications are correlated with subsequent development of coronary artery calcifications, but their aetiology is predominantly different. Eur J Radiol 2007;63(3):396-400.

25. Topal U, Kaderli A, Topal NB, et al. Relationship between the arterial calcification detected in mammography and coronary artery disease. Eur J Radiol 2007 Sep;63(3): 391-395.

26. Kemmeren JM, Beijerinck D, van-Noord PA, et al. Breast arterial calcifications: association with diabetes mellitus and cardiovascular mortality. Radiology 1996;201: 75-78 (4).

27. Nasir K, McEvoy JW. Recognizing breast arterial calcification as atherosclerotic CVD equivalent. From evidence to action. J Am Coll Cardiol Img 2016;9(4):361-363.

28. Elisaf M. The treatment of coronary heart disease: an update. Part 1: an overview of the risk factors for cardiovascular disease. Curr Med Res Opin 2001;17:18-26.

29. John JM, Bhatt DL. Emerging risk factors for atherosclerosis. Indian Heart J 2007;59: $28-37$. 